\title{
NOUVELLE
}

\section{Les nanotubes bactériens, acteurs de la compétition entre Bacillus subtilis et Bacillus megaterium}

Sophia Belkhir, Valentine Marion
École normale supérieure de Lyon, Département de biologie, Master biologie, Lyon, France.

sophia.belkhir@ens-lyon.fr valentine.marion@ens-lyon.fr
> Les bactéries peuvent vivre en communauté, dont la structure est régulée par de nombreuses interactions biotiques et abiotiques. Les interactions entre bactéries peuvent résulter en une diminution de valeur sélective dans des cas de prédation, de parasitisme, ou de lutte impliquant des toxines. De même, lorsque plusieurs espèces bactériennes partagent une niche écologique, cela peut entraîner des situations de compétition pour l'espace ou les ressources. Au contraire, des comportements collaboratifs entre espèces bactériennes peuvent augmenter le succès reproducteur de chacun des partenaires [1]. L'organisation en communauté nécessite une communication entre les bactéries impliquées. Chaque bactérie possède des structures lui permettant d'interagir avec les autres, en établissant ou non des contacts directs. En l'absence de contact direct, la communication peut notamment se faire en utilisant des signaux moléculaires qui diffusent dans le milieu. C'est l'exemple du quorum sensing, un mécanisme de synchronisation de l'expression ou de la répression de gènes particuliers au sein d'une population bactérienne et en fonction de la densité de cette population [2]. Dans le cas d'interactions avec des contacts directs, des structures particulières font entrer en contact les membranes ou les parois des deux bactéries. Certaines structures, telles que les pilis ou les systèmes de sécrétion, sont déjà bien connues. Les systèmes de sécrétion de type III, IV et VI permettent, par exemple, I'injection d'effecteurs protéiques direc- tement dans des cellules cibles à travers le système pariétal bactérien [3]. Plus récemment, d'autres structures permettant des interactions cellule-cellule ont été découvertes: les nanotubes. II s'agit de protrusions cytoplasmiques tubulaires qui ont d'abord été découvertes chez les cellules eucaryotes [4]. Chez ces cellules, les nanotubes permettent de faciliter le transport intercellulaire de molécules cytoplasmiques, d'organelles et même de virus [5]. En 2011, Dubey et Ben-Yehuda ont montré que ces structures existent aussi chez les procaryotes [6]. Leur étude sur Bacillus subtilis a prouvé que ces bactéries Gram-positives sont capables de former des nanotubes entre bactéries de la même espèce ou avec des bactéries d'espèce différente pour échanger des molécules cytoplasmiques, dont des plasmides. Chez ces bactéries, la phosphodiestérase $\mathrm{ymdB}$ contribue à la formation des nanotubes à partir de la membrane plasmique [7]. Plus récemment, Stempler et al. [8] ont mis en évidence l'échange de toxines et de nutriments via des nanotubes entre deux souches Gram-positives, B. subtilis et $B$. megaterium, et ont cherché à préciser le fonctionnement et les conséquences de cette interaction.

\section{Les nanotubes permettent}

de délivrer directement des toxines dans les bactéries ciblées

Le système toxine-antitoxine est un système d'attaque ou de défense très répandu chez les bactéries. Le système consiste en deux composantes: une toxine stable de type protéique dont la surexpression tue les bactéries, et une antitoxine instable qui neutralise l'action de la toxine dans la bactérie qui la produit. Les bactéries peuvent donc produire des toxines qui leur sont utiles dans le cadre d'une relation de compétition. Les toxines peuvent être libérées dans le milieu, ou bien délivrées directement à la cellule cible. Stempler et al. [8] ont montré que $B$. subtilis utilise le couple de toxine-antitoxine WapA/Wapl pour inhiber la croissance de $B$. megaterium. En effet, en co-cultivant les deux souches bactériennes, les auteurs ont remarqué que la croissance de $B$. megaterium est inhibée mais que celle de $B$. subtilis reste semblable à la croissance observée lorsque cette souche est cultivée seule. Une deuxième expérience de co-culture a été réalisée en présence d'une souche de $B$. subtilis incapable de produire la protéine WapA et d'une souche sauvage de $B$. megaterium. La croissance de cette dernière n'est alors plus inhibée et est semblable à celle en culture seule. Ainsi, la production de la protéine WapA par B. subtilis est nécessaire pour inhiber la croissance de $B$. megaterium.

Pour déterminer si cette inhibition est dépendante d'une interaction directe ou non, les auteurs ont utilisé une chambre de Transwell, montage composé d'un compartiment inférieur séparé du compartiment supérieur par une membrane comportant des pores de 0,4 $\mu \mathrm{m}$ de diamètre. Ainsi, les bactéries ne peuvent pas traverser, et seules les petites molécules contenues dans le milieu, comme les toxines, peuvent passer d'un compartiment à l'autre. B. subtilis est placée 
A

Préculture

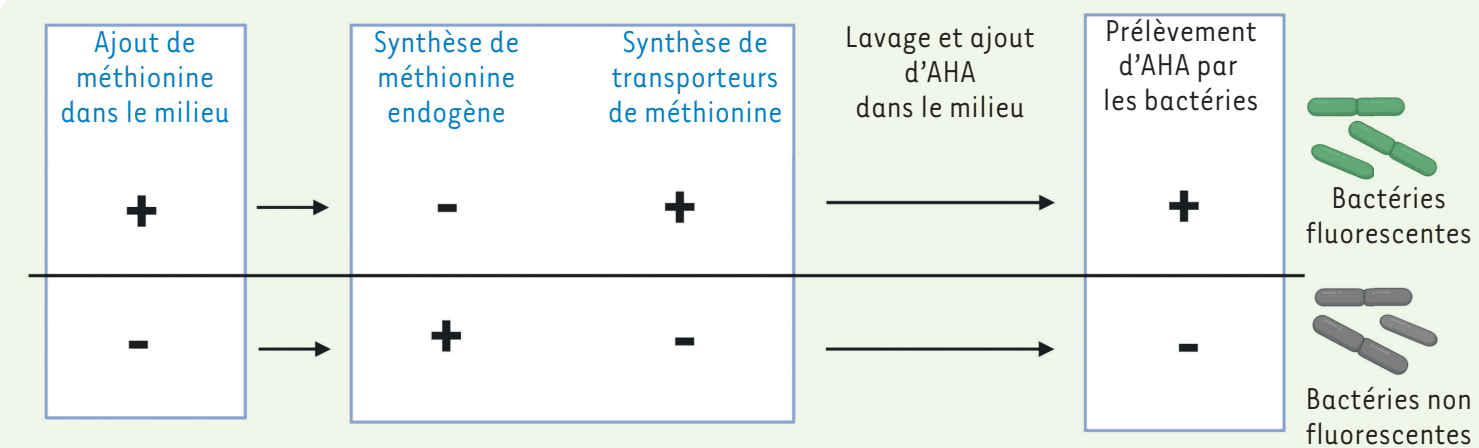

B

B. subtilis auxotrophe à la méthionine

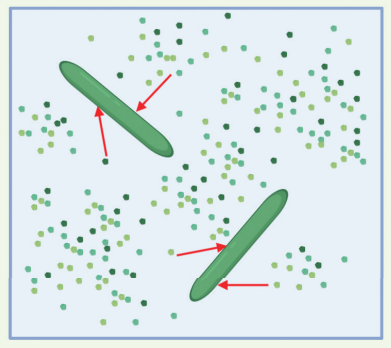

C B. subtilis auxotrophe à la méthionine $+B$. megaterium

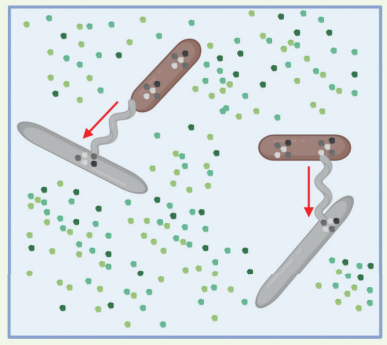

Légende :

$\begin{array}{ll}\therefore & \text { AHA } \\ \therefore & \text { Méthionine endogène } \\ & \text { Nanotube } \\ \longrightarrow & \text { Flux de nutriments } \\ & \text { B. megaterium } \\ & \text { B. subtilis non fluorescente } \\ & \text { B. subtilis fluorescente }\end{array}$

Figure 1. Dispositif expérimental permettant de montrer que Bacillus subtilis prélève la méthionine de Bacillus megaterium. (A) عffet de la méthionine exogène sur la synthèse de méthionine endogène et de transporteurs de méthionine par les bactéries. Une pré-culture en présence de méthionine exogène conduit au prélèvement de la L-azidohomoalanine ( $A H A)$ fluorescente, dans le milieu par les bactéries, alors qu'une préculture sans méthionine exogène restreint le prélèvement d’AHA par les bactéries. (B-C) B. subtilis prélève de la méthionine chez $B$. megaterium. (B) $B$. subtilis auxotrophe à la méthionine pré-cultivée en présence de méthionine et mise seule dans un milieu contenant de l'AHA. (C) B. subtilis auxotrophe à la méthionine pré-cultivée en présence de méthionine et mise en contact avec $B$. megaterium capable de produire sa méthionine.

dans un compartiment et $B$. megaterium dans l'autre. Après incubation, la croissance de $B$. megaterium n'est pas inhibée, ce qui signifie que l'inhibition de B. megaterium par B. subtilis est dépendante d'un contact direct. WapA n'est donc pas sécrétée dans le milieu mais bien délivrée directement d'une bactérie à l'autre.

Pour aller plus loin, Stempler et al. ont émis l'hypothèse que le contact entre les bactéries pourrait se faire via des nanotubes. Pour tester cette hypothèse, les auteurs ont réalisé des co-cultures de $B$. megaterium sauvage avec $B$. subtilis mutée ne synthétisant plus la phosphodiestérase $\mathrm{Ymd} B$, protéine nécessaire à la formation des nanotubes [7]. $B$. megaterium croît alors normalement. La protéine $\mathrm{YmdB}$ est donc nécessaire à l'inhibition de B. megaterium par $B$. subtilis. Pour confirmer l'hypothèse selon laquelle $B$. subtilis formerait des nanotubes la reliant à $B$. megaterium, via lesquels elle délivrerait la toxine WapA, les auteurs ont alors utilisé une technique de microscopie électronique à haute résolution couplée à un marquage immuno-gold de WapA. Les observations microscopiques permettent de montrer que certaines $B$. subtilis sont capables de former des nanotubes avec $B$. megaterium. Les résultats obtenus permettent également de visualiser la protéine WapA associée aux nanotubes. Cela suggère que, par le biais des nanotubes, la protéine WapA transiterait jusqu'au cytoplasme de $B$. megaterium. Cependant, à défaut d'une précision microscopique suffisante, la localisa- tion exacte de la protéine WapA par rapport au nanotube n'est pas encore connue, et il n'est pas possible de savoir si WapA se déplace à l'intérieur ou à la surface du nanotube.

Ainsi, les nanotubes peuvent être utilisés pour délivrer des toxines directement dans les bactéries voisines, conduisant à la mort de celles-ci. L'étude de Stempler et al. s'est ensuite intéressée au potentiel rôle des nanotubes dans le prélèvement de ressources nutritives.

\section{Les nanotubes permettent de prélever} directement des nutriments chez les bactéries ciblées

Dans certains milieux, les bactéries sont en compétition pour l'accès aux ressources. Certaines prélèvent directement les nutriments dans leur milieu, 
alors que d'autres ont développé des structures originales pour accéder à des nutriments à plus longue distance. Par exemple, Delftia sp. produit des extensions cytoplasmiques appelées «nanopodes » qui permettent de projeter à longue distance des vésicules de membrane externe contenant des enzymes dégradant les nutriments, ce qui favorise leur absorption par la bactérie [9]. En principe, lorsqu'un un nutriment tel qu'un acide aminé est présent en abondance dans un milieu, il est moins coûteux pour une bactérie de synthétiser des transporteurs pour le prélever que de le produire elle-même. Inversement, si un nutriment est peu présent dans un milieu, cela peut entraîner l'activation de ses voies de synthèse. Afin de visualiser l'assimilation et l'échange de méthionine par $B$. subtilis et $B$. megaterium en culture, Stempler et al. ont développé un système rapporteur dans lequel les bactéries sont cultivées en présence d'un analogue de la méthionine, la L-azidohomoalanine ( $A H A)$, couplé à un fluorophore. Cela permet de visualiser les bactéries fluorescentes qui ont prélevé I'AHA du milieu pour assurer leur métabolisme (Figure 1A). Les auteurs ont développé une souche de B. subtilis auxotrophe à la méthionine, c'est-à-dire incapable de synthétiser la méthionine, et étudié comment celle-ci se procurait cet acide aminé. Suivant le principe exposé précédemment, les bactéries pré-cultivées dans un milieu riche en méthionine ont tendance à synthétiser des transporteurs, et lorsqu'elles sont cultivées en présence d'AHA, à le prélever directement du milieu, elles apparaissent donc fluorescentes (Figure lB). En revanche, mises en contact avec $B$. megaterium produisant de la méthionine, ces mêmes bactéries B. subtilis n'apparaissent plus fluorescentes, mais sont toujours vivantes (Figure IC). Elles n'ont pas prélevé d'AHA du milieu, mais se sont procurées de la méthionine d'une autre façon. Cela suggère que $B$. subtilis est capable de puiser directement de la méthionine chez les $B$. megaterium environnantes, qui sont la seule source de méthionine non fluorescente du milieu. Les auteurs ont ensuite constaté par l'intermédiaire d'un dispositif de Transwell que ce phénomène était bien dépendant d'un contact direct. De plus, les bactéries B. subtilis n'exprimant pas la phosphodiestérase $\mathrm{ymdB}$ ne sont plus capables de se procurer la méthionine à partir de $B$. megaterium, et se contentent de prélever l'AHA du milieu. Puisque $\mathrm{YmdB}_{\mathrm{m}}$ est nécessaire à la formation des nanotubes $[7]$, cela suggère que ce sont les nanotubes qui permettent de relier $B$. subtilis à sa rivale de niche $B$. megaterium, et qui font circuler les nutriments présents dans le cytoplasme de B. megaterium. L'étude de Stempler et al. a donc démontré que $B$. subtilis est capable de prélever des nutriments de $B$. megaterium par le biais de nanotubes. Ainsi, dans certains milieux, la capacité de B. subtilis à produire des nanotubes la connectant à des bactéries d'autres espèces partageant sa niche peut être un avantage et lui conférer une valeur sélective plus élevée.

\section{Conclusion}

L'étude de Stempler et al. souligne donc l'importance des nanotubes, et montre que leur implication dans la compétition inter-espèces se fait de deux façons: à la fois en permettant à $B$. subtilis de délivrer des toxines directement dans le cytoplasme de $B$. megaterium, et en permettant à $B$. subtilis de puiser des nutriments chez B. megaterium (Figure 2). Les auteurs ont par ailleurs montré que les deux phénomènes sont indépendants. Ainsi, au sein des nanotubes, des molécules peuvent circuler indépendamment dans les deux sens, depuis la bactérie donneuse vers la bactérie receveuse ou inversement. Le contrôle de la directionnalité reste méconnu. II pourrait exister des nanotubes distincts qui assurent chacun la circulation dans des sens opposés, ou bien des nanotubes bidirectionnels, dans lesquels le sens de circulation dépendrait du cargo, et par exemple, de la taille de celui-ci. La production d'extensions cytoplasmiques sous forme de nanotubes pour délivrer ou prélever des molécules peut paraître coûteuse pour les bactéries, mais les nanotubes ont pour intérêt de permettre de cibler directement le cytoplasme de la bactérie avec laquelle se déroule l'échange de molécules. Cela est plus efficace que les transferts passant par le milieu extracellulaire et impliquant la diffusion de toxines ou de métabolites, car les molécules échangées risquent de se disperser et de ne pas atteindre leur cible, ou d'être captées par d'autres bactéries.

La présence de nanotubes a été montrée chez de nombreuses espèces bactériennes, tant Gram-positives que Gram-négatives [10], suggérant qu'il s'agit d'une structure conservée. Selon le type de molécule échangée et la directionnalité de l'échange, la présence de nanotubes peut donc facilement donner lieu à une relation de compétition interspécifique, et peut permettre à une espèce d'exploiter au maximum les ressources de sa niche potentielle. Dans des conditions carencées, posséder des nanotubes qui inhibent la croissance des bactéries voisines peut alors donner un avantage et permettre aux bactéries concernées de réussir à dominer la niche. Un tel mécanisme peut notamment être profitable à des espèces en compétition pour un même territoire dans des structures multicellulaires de type biofilm.

Même si l'article de Stempler et al. présente une situation de compétition entre bactéries, les nanotubes servent aussi à la collaboration entre les bactéries. Par exemple, Escherichia coli peut produire des nanotubes et partager des nutriments cytoplasmiques avec des bactéries voisines d'autres espèces comme Acinetobacter baylili, et ainsi procéder à une nutrition croisée (crossfeeding) [11]. Les nanotubes peuvent également être utilisés pour transférer de l'information, notamment génétique. 


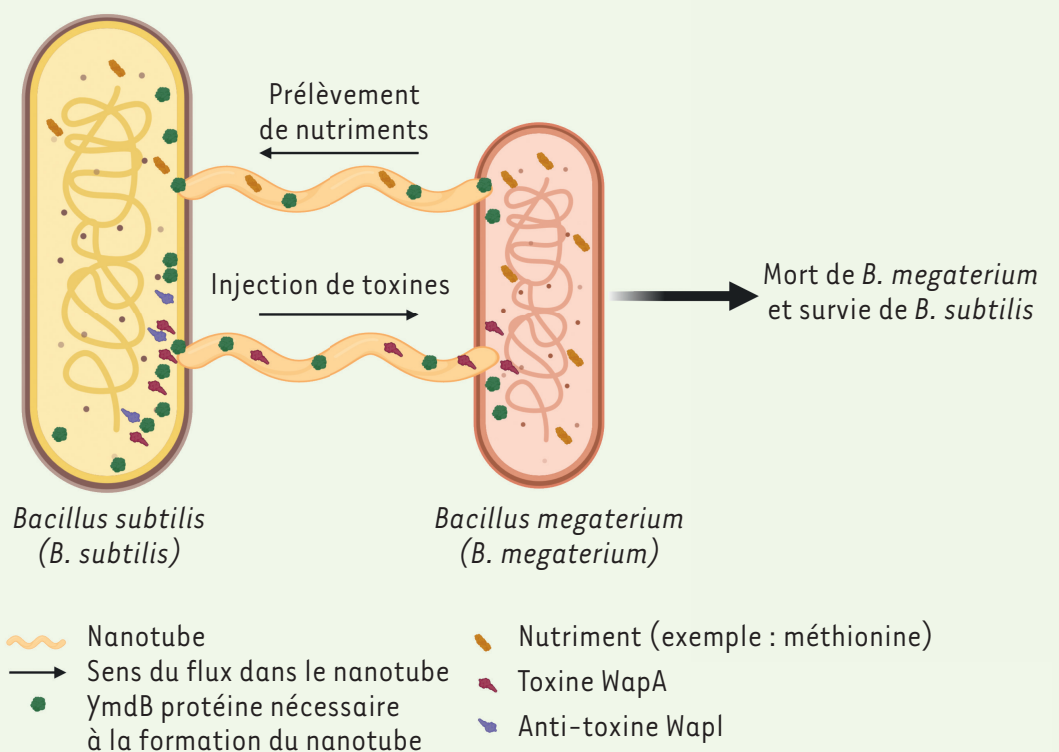

Figure 2. Bacillus subtilis injecte des toxines et extrait des nutriments via des nanotubes dans le cadre d'une relation de compétition avec Bacillus megaterium. Les nanotubes connectent les cytoplasmes des deux bactéries et leur formation nécessite la phosphodiestérase $\mathrm{YmdB}$. Les flèches représentent le sens du flux dans le nanotube, ici un nanotube permet l'injection de la toxine WapA depuis $B$. subtilis vers le cytoplasme de $B$. megaterium et un autre nanotube permet de puiser les nutriments de cette $B$. megaterium et de les acheminer vers $B$. subtilis.

Dubey et al. ont en effet mis en évidence l'échange de plasmides non conjugatifs entre $B$. subtilis et d'autres espèces éloignées évolutivement [8]. Plus récemment, des chercheurs ont identifié un nouveau type de nanotubes, appelés nanowires, notamment présents chez Shewanella oneidensis, qui permettent l'échange d'électrons par le biais de membranes possédant des complexes d'oxydo-réduction [12]. Ce sont autant d'exemples soulevant l'importance des nanotubes dans la communication entre microorganismes.
Il est donc primordial d'étudier le fonctionnement des nanotubes pour comprendre la communication entre bactéries, notamment d'espèces différentes, et l'organisation des individus en communautés. En effet, l'établissement de structures multicellulaires telles que les biofilms, ou encore le transfert de plasmides de résistance entre bactéries sont des problématiques importantes dans le monde médical, et nous avons vu que ces mécanismes peuvent reposer sur la formation de nanotubes. Des perspectives intéressantes pourraient alors être envisagées en inhibant ou détournant le fonctionnement de nanotubes qui contribueraient à la virulence de certaines espèces, ou à une communication entre pathogènes qui serait néfaste pour l'hôte. $\diamond$

Bacterial nanotubes play a key role in the competition between Bacillus subtilis and Bacillus megaterium

\section{LIENS D'INTÉRÊT}

Les auteures déclarent n'avoir aucun lien d'intérêt concernant les données publiées dans cet article.

\section{RÉFÉRENCES}

1. Little AEF, Robinson CJ, Peterson SB, et al. Rules of engagement: interspecies interactions that regulate microbial communities. Annu Rev Microbiol 2008 ; 62 : 375-401.

2. Ng WL, Bassler BL. Bacterial quorum-sensing network architectures. Annu Rev Genet 2009 ; 43 : 197-222.

3. Green $\varepsilon R$, Mecsas J. Bacterial secretion systems: an overview. Microbiol Spectr 2016 ; 4.

4. Ramírez-Weber FA, Kornberg TB. Cytonemes: cellular processes that project to the principal signaling center in drosophila imaginal discs. Cell $1999 ; 97$ : 599-607.

5. Abounit S, Zurzolo C. Wiring through tunneling nanotubes: from electrical signals to organelle transfer. J Cell Sci 2012 ; 125 : 1089-98.

6. dubey gp, ben-yehuda s. intercellular nanotubes mediate bacterial communication. Cell 2011 ; 144 : 590-600.

7. Dubey GP, Malli Mohan GB, Dubrovsky A, et al. architecture and characteristics of bacterial nanotubes. Dev Cell 2016 ; 36 : 453-61.

8. Stempler 0 , Baidya AK, Bhattacharya S, et al. Interspecies nutrient extraction and toxin delivery between bacteria. Nat Commun $2017 ; 8$.

9. Shetty A, Chen S, Tocheva El, et al. Nanopods: a new bacterial structure and mechanism for deployment of outer membrane vesicles. PLoS One 2011; 6 .

10. Baidya AK, Bhattacharya S, Dubey GP, et al. Bacterial nanotubes: a conduit for intercellular molecular trade. Curr Opin Microbiol $2018 ; 42$ : 1-6.

11. Pande $S$, Shitut $S$, Freund L, et al. Metabolic crossfeeding via intercellular nanotubes among bacteria. Nat Commun $2015 ; 6: 6238$.

12. Pirbadian S, Barchinger SE, Leung KM, et al. Shewanella oneidensis MR-1 nanowires are outer membrane and periplasmic extensions of the extracellular electron transport components. Proc Natl Acad Sci USA 2014 ; 111 : 12883-8.

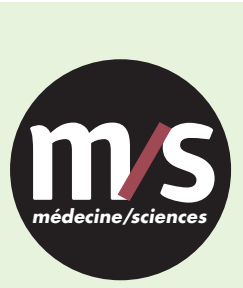

\section{Tarifs d'abonnement $\mathrm{m} / \mathrm{s}-\mathbf{2 0 2 0}$ \\ Abonnez-vous \\ à médecine/sciences}

> Grâce à $\mathrm{m} / \mathrm{s}$, vivez en direct les progrès des sciences biologiques et médicales

Bulletin d'abonnement page 1098 dans ce numéro de $\mathrm{m} / \mathrm{s}$

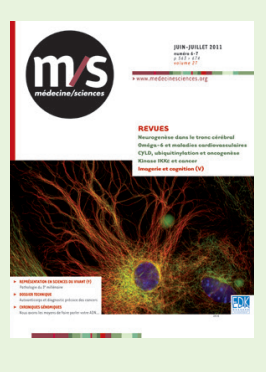

